\title{
Mesenteric ischemia following the correction of adult spinal deformity: case report
}

\author{
Krishn Khanna, MD, and Sigurd H. Berven, MD \\ Department of Orthopaedic Surgery, University of California, San Francisco, California
}

\begin{abstract}
Vascular complications are an important adverse event that can be associated with spinal reconstructive surgery. Direct injury of vessels, or indirect traction or compression of vessels, can cause both arterial and venous injury. Indirect compression of the mesenteric vessels is a well-recognized complication of bracing and surgical care of children with spinal deformity (superior mesenteric artery syndrome), but the complication is not common or well recognized in the adult population with spinal deformity. The purpose of this case report is to detail the case of postoperative mesenteric ischemia in a 63-year-old man in whom a posterior approach was used to perform spinal deformity correction. Preoperatively, the patient had had significant lumbar hypolordosis. The reconstructive surgery with the use of posterior-based osteotomies resulted in a shortening of the posterior column of the spine but a relative lengthening of structures anterior to the spine. The significant lordosis achieved by the surgery led to an acute worsening of the mesenteric stenosis suffered by the patient. He required a vascular surgery intervention to restore perfusion to the bowel. Recognition of severe vasculopathy is important in anticipating potential postoperative vascular insufficiency. This case report will inform surgeons and clinicians to have a higher index of suspicion for the exacerbation of vascular insufficiency, including mesenteric pathology, in patients undergoing surgery that involves significant realignment of the spine. Preoperative recognition of vascular insufficiency and treatment of symptomatic disease may limit the occurrence of postoperative vascular complications in spinal reconstructive surgery.

https://thejns.org/doi/abs/10.3171/2016.8.SPINE16571
\end{abstract}

KEY WORDS adult spinal deformity; mesenteric ischemia; vascular complications; lumbar

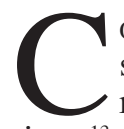

OMPLICATION rates following adult spinal deformity surgery are high, ${ }^{14}$ with the most recent literature reporting nearly a $70 \%$ incidence of complications. ${ }^{13}$ Preoperative vascular disease is a significant independent predictor of postoperative complications in patients undergoing spine surgery. ${ }^{1,10}$ In anterior approaches to the spine, vascular injury can result from direct injury to the artery or vein that occurs when manipulating the arteries and veins during exposure, with a reported incidence of approximately 3\%? ? $^{7}$ Posterior approaches have also resulted in vascular complications, especially those that entail correcting deformities associated with chronic or systemic comorbidities. ${ }^{12,16}$ Indirect compromise of circulation is well recognized in bracing and deformity correction in children with scoliosis. $5,6,8,9,11$ The purpose of this case report is to highlight the risk of vascular compromise in posterior-based realignment procedures of the spine and to inform patients and surgeons of this risk to guide an informed choice regarding options for care.
We describe the case of an adult patient who underwent scoliosis correction that was complicated by vascular compromise of his abdominal circulation. Despite a preoperative vascular surgery consultation suggesting that the patient was at low risk for exacerbation of his known mesenteric artery disease, the patient still went on to develop mesenteric ischemia, which was associated with significant malnutrition, wound complications, the need for endovascular stenting, and a significantly lengthened hospital stay. Recognition of the impact of deformity correction on the tension of great vessels is important in anticipating potential complications in deformity surgery.

\section{Case Report}

History

A 63-year-old man, with a history of testicular cancer treated with radiotherapy and radical lymph node dissection, presented to our clinic complaining of 2 years of pro- 

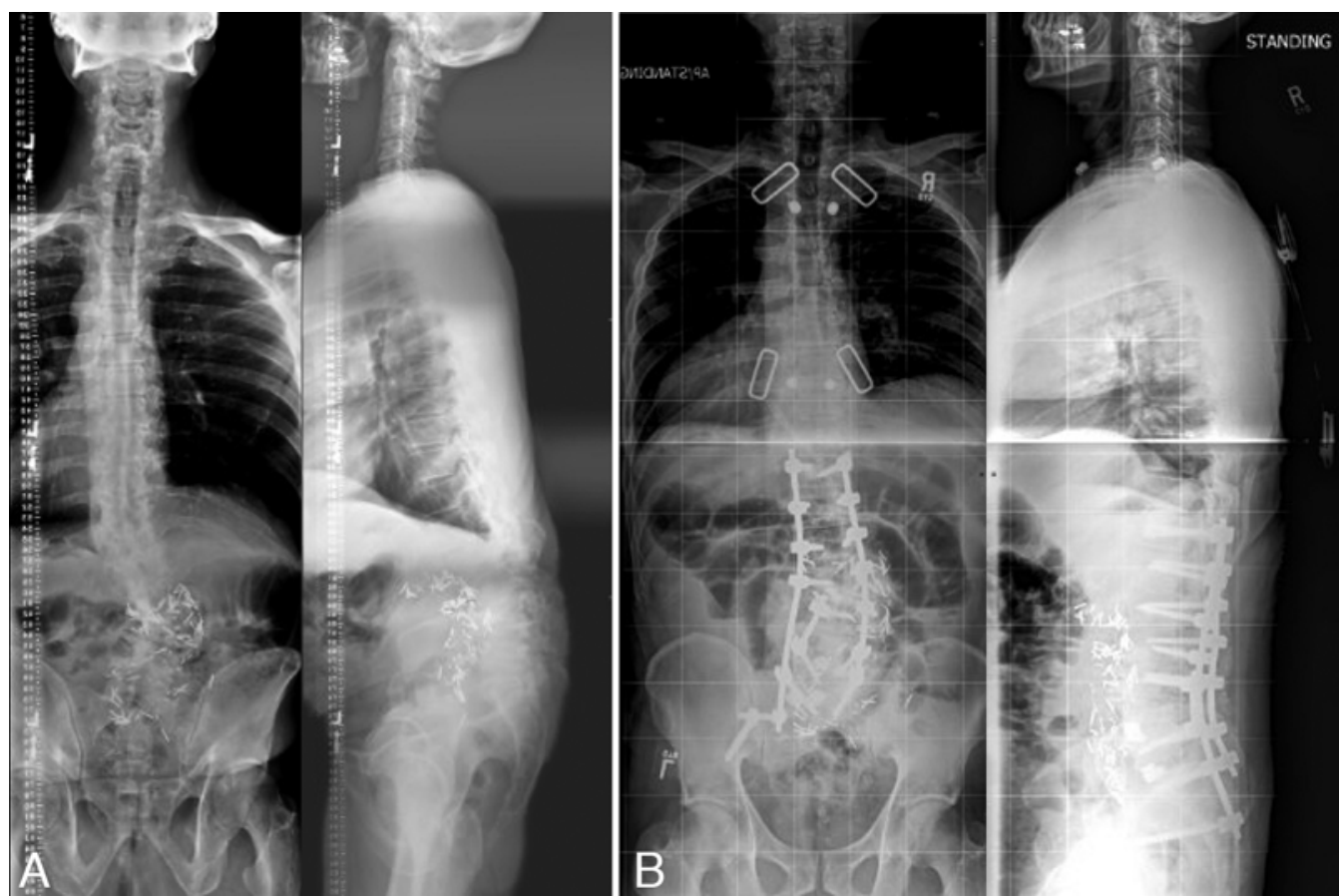

FIG. 1. Preoperative (A) and postoperative (B) scoliosis radiographs demonstrating the change in morphology achieved by the surgery. Preoperatively, the patient had severe degenerative lumbar dextroscoliosis and kyphosis of the lumbar spine centered at L-3, with a global SVA of $12.5 \mathrm{~cm}$. He underwent a transforaminal lumbar interbody fusion at L3-4, L4-5, and L5-S1; an L-4 pedicle subtraction osteotomy; and a T10-ilium posterior spinal fusion. The patient regained significant lumbar lordosis and his global SVA was $4 \mathrm{~cm}$.

gressive low-back pain, right-sided radicular symptoms, and a longstanding right foot drop. Of note, he indicated that he had had diarrhea for 3 months and had lost approximately 30 pounds during that time. The issue of weight loss had been looked into by the patient's primary care physician, who ordered laboratory testing and colonoscopy and who attributed the weight loss to diverticulosis and psychosocial stress from the recent death of a family member. The patient's symptoms were severely impacting his quality of life, and after a thorough discussion of the high rate of complications associated with adult spinal deformity correction, especially given his surgical history, the patient consented to the procedure.

\section{Examination and Imaging}

The results of the examination were notable for numbness in the L-4 and L-5 distribution. Radiography showed severe degenerative lumbar dextroscoliosis and kyphosis of the lumbar spine centered at L-3, with a global sagittal vertical axis (SVA) of $12.5 \mathrm{~cm}$. Also evident were innumerable surgical clips from the patient's previous abdominal lymph node dissection. To evaluate the possibility of an anterior approach given the severe calcification of the patient's arteries and history of extensive retroperitoneal surgery and radiotherapy, CT angiography (CTA) of the abdomen and pelvis was performed and showed significant atherosclerosis of the anterior vessels. Notably, the celiac artery was $60 \%$ stenosed and the superior mesenteric artery (SMA) was 80\%-90\% stenosed (Fig. 1). Consultation with a vascular surgeon indicated that an anterior approach would be too risky, given the prior anterior surgical dissection. A posterior-only surgical approach was planned.

\section{Operation}

The patient underwent a transforaminal lumbar interbody fusion at L3-4, L4-5, and L5-S1; an L-4 pedicle subtraction osteotomy; and a T10-ilium posterior fusion. He regained significant lumbar lordosis and his global SVA was $4 \mathrm{~cm}$. The patient fared well postoperatively with no neurological changes and was discharged on postoperative Day 5.

\section{Postoperative Course}

One week after discharge, the patient was admitted to an outside facility for postprandial abdominal pain, an inability to tolerate any oral intake, and a 20-pound weight loss since surgery. Abdominal CTA was diagnostic for mesenteric ischemia, with critical stenosis of the SMA and celiac artery (Fig. 2). He was placed on total parenteral nutrition. He was also found to have drainage from his wound and leukocytosis greater than 20,000 cells $/ \mu l$. Three spine wound washouts were performed, with plastic surgery assisting for the final closure. The patient's stay was complicated by an acute kidney injury that developed secondary to poor oral intake and numerous contrast boluses. Once his creatinine level normalized, the patient underwent endovascular stenting of the celiac artery and SMA. He was able to tolerate some amount of pureed food postoperatively and was discharged 3 weeks after his readmission. Repeat CTA was performed because the pa- 

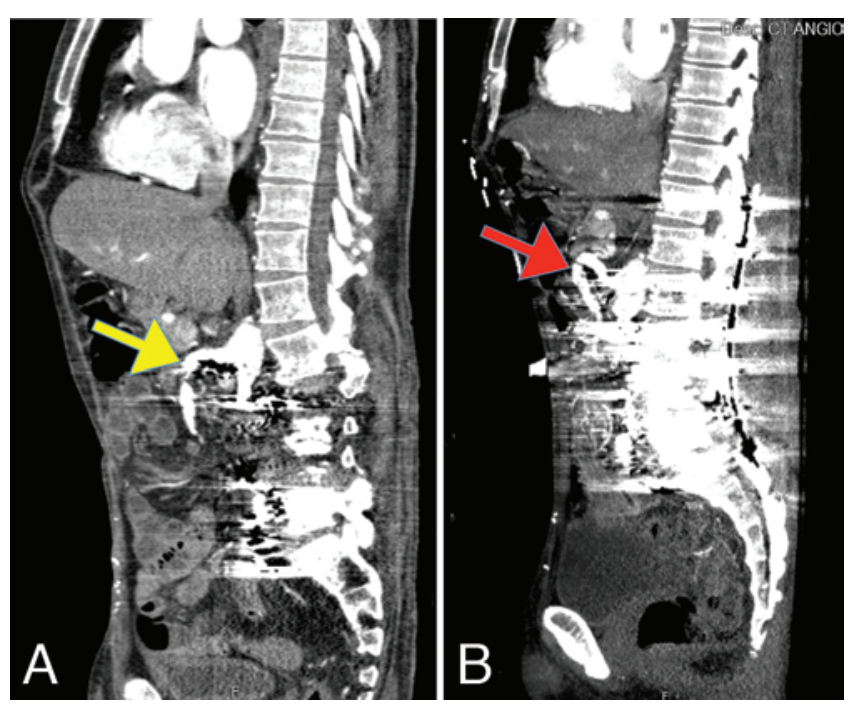

FIG. 2. A: Preoperative CT angiogram of the abdomen demonstrating the SMA take-off at nearly a right angle from the aorta (arrow). B: Postoperative CT angiogram of the abdomen demonstrating the large correction of the adult deformity and resulting lordosis, as well as the consequent acute angle at which the SMA takes off from the aorta (arrow). Figure is available in color online only.

tient was slow to tolerate an oral intake of food, and the studies demonstrated patent mesenteric arteries without high-grade stenosis.

\section{Discussion}

Abdominal complications following scoliosis surgery have been reported since the earliest days of scoliosis correction in pediatric patients. SMA syndrome is a wellknown complication from the treatment of children with scoliosis, ${ }^{5,8,9}$ forming part of a larger constellation of complications called "cast syndrome." Hyperlordosis, which results from casting or surgical correction, changes the take-off angle of the mesenteric arteries from the aorta, most notably the SMA, which drapes over the third portion of the duodenum. This more acute angle causes duodenal compression and leads to an extraluminal small bowel obstruction. ${ }^{2,11}$ More recent case reports have also described acute compartment syndrome as a complication of a lordotic scoliosis correction. ${ }^{3}$ To the best of our knowledge, ours is the first known report of mesenteric ischemia in a patient who underwent adult spinal deformity correction.

Different techniques for creating lumbar lordosis may present different risks to a patient undergoing adult spinal deformity correction. The anterior approach involves risk of direct injury to anterior vasculature and also elongates the anterior column of the spine.? Posterior approaches, on the flip side, shorten the spinal column. The axis of rotation around which this shortening and lordosis occur varies based on the types of osteotomies. Specifically, the Smith-Peterson osteotomy hinges on the posterior longitudinal ligament and distracts the anterior column, ${ }^{15}$ whereas the pedicle subtraction osteotomy hinges on the anterior longitudinal ligament and distracts the vessels anterior to the spine. ${ }^{17}$
We suspect that a similar pathomechanism to that seen in cast syndrome led to the patient's mesenteric ischemia. Preoperative angiography showed stenosis of the mesenteric vessels. This patient underwent an L-4 pedicle subtraction osteotomy. The regained lumbar lordosis distracted the tissue, in this case vasculature, lying anterior to the axis of correction and led to a critical stenosis of the patient's mesenteric arteries and the complete intolerance of oral intake. We believe the nutritional deficits caused by the mesenteric ischemia and poor oral intake postoperatively contributed to the patient's wound dehiscence and infection. The anatomy of the aorta has been shown to be altered after scoliosis surgery. ${ }^{4}$ This is clearly demonstrated on the pre- and postoperative images of the SMA's take-off from the aorta (Fig. 2). Along with the additional plaque burden seen in patients with adult degenerative scoliosis, ${ }^{2}$ this altered anatomy may have been responsible for the acute worsening of the patient's mesenteric ischemia. In hindsight, there were aspects of the patient's history that should have raised suspicion for tenuous mesenteric circulation. The patient reported several months of progressing diarrhea and a 30-pound weight loss. However, preoperatively, he was able to tolerate solid oral intake.

The patient underwent CTA as well as a vasculature surgery consultation prior to the deformity correction. This was requested partially to consider the feasibility of an anterior approach ${ }^{7}$ but also to evaluate the risk to the highly calcified abdominal aorta when reestablishing lordosis of the spine. However, there was little consideration given to the mesenteric circulation and how the deformity correction may impact it.

\section{Conclusions}

The present report aims to describe a case of mesenteric ischemia that presented after sagittal deformity correction in an adult with vascular disease and a history of abdominal surgery and radiotherapy. This is a complication that should be considered when planning a sagittal deformity correction procedure in a patient with highly calcified and stenotic anterior vasculature. Having a higher index of suspicion for the exacerbation of this mesenteric pathology could allow surgeons to address it preoperatively with medical or endovascular intervention and minimize postoperative complications in adult spinal deformity surgery.

\section{References}

1. Abdul-Jabbar A, Berven SH, Hu SS, Chou D, Mummaneni PV, Takemoto S, et al: Surgical site infections in spine surgery: identification of microbiologic and surgical characteristics in 239 cases. Spine (Phila Pa 1976) 38:E1425-E1431, 2013

2. Ayça B, Rakıcı T, Atıcı Y, Avsar M, Yuksel Y, Akın F, et al: Adult degenerative scoliosis associated with increased aortic diameter and plaque burden and composition. Vascular 24:315-322, 2016

3. Boniello A, Verma K, Sees JP, Miller F, Dabney K: Delayed abdominal compartment syndrome as a complication of spinal surgery: literature review and case report. Spine Deform 1:464-467, 2013

4. Bullmann V, Fallenberg EM, Meier N, Fischbach R, Lerner $\mathrm{T}$, Schulte TL, et al: The position of the aorta relative to the 
spine before and after anterior instrumentation in right thoracic scoliosis. Spine (Phila Pa 1976) 31:1706-1713, 2006

5. Crowther MAA, Webb PJ, Eyre-Brook IA: Superior mesenteric artery syndrome following surgery for scoliosis. Spine (Phila Pa 1976) 27:E528-E533, 2002

6. Dorph MH: The cast syndrome-review of the literature and report of a case. N Engl J Med 243:440-442, 1950

7. Fantini GA, Pappou IP, Girardi FP, Sandhu HS, Cammisa FP Jr: Major vascular injury during anterior lumbar spinal surgery: incidence, risk factors, and management. Spine (Phila Pa 1976) 32:2751-2758, 2007

8. Feneley RC, Macdonald D: Acute arteriomesenteric duodenal ileus as a complication following operative correction of scoliosis. Postgrad Med J 45:781-784, 1969

9. Lam DJL, Lee JZJ, Chua JHY, Lee YT, Lim KBL: Superior mesenteric artery syndrome following surgery for adolescent idiopathic scoliosis: a case series, review of the literature, and an algorithm for management. J Pediatr Orthop B 23:312-318, 2014

10. Schairer WW, Carrer A, Deviren V, Hu SS, Takemoto S, Mummaneni $P$, et al: Hospital readmission after spine fusion for adult spinal deformity. Spine (Phila Pa 1976) 38:16811689,2013

11. Shah MA, Albright MB, Vogt MT, Moreland MS: Superior mesenteric artery syndrome in scoliosis surgery: weight percentile for height as an indicator of risk. J Pediatr Orthop 23:665-668, 2003

12. Shih P, Slimack NP, Roy A, Fessler RG, Koski TR: Abdominal complications following posterior spinal fusion in patients with previous abdominal surgeries. Neurosurg Focus 31(4):E16, 2011

13. Smith JS, Klineberg E, Lafage V, Shaffrey CI, Schwab F, Lafage R, et al: Prospective multicenter assessment of perioperative and minimum 2-year postoperative complication rates associated with adult spinal deformity surgery. J Neurosurg Spine 25:1-14, 2016

14. Smith JS, Shaffrey CI, Glassman SD, Berven SH, Schwab FJ, Hamill CL, et al: Risk-benefit assessment of surgery for adult scoliosis: an analysis based on patient age. Spine (Phila Pa 1976) 36:817-824, 2011

15. Smith-Petersen MN, Larson CB, Aufranc OE: Osteotomy of the spine for correction of flexion deformity in rheumatoid arthritis. J Bone Jt Surg Am 27:1-11, 1945

16. Sugrue PA, O'Shaughnessy BA, Nasr F, Koski TR, Ondra SL: Abdominal complications following kyphosis correction in ankylosing spondylitis. J Neurosurg Spine 10:154-159, 2009

17. Wang MY, Berven SH: Lumbar pedicle subtraction osteotomy. Neurosurgery 60 (2 Suppl 1):ONS140-ONS146, 2007

\section{Disclosures}

Dr. Berven reports being a consultant for Medtronic, Stryker, Globus Medical, and RTI; he holds patents with Medtronic, Stryker, and CoorsTek Medical.

\section{Author Contributions}

Analysis and interpretation of data: Khanna. Drafting the article: Khanna. Critically revising the article: Berven. Reviewed submitted version of manuscript: Berven. Approved the final version of the manuscript on behalf of both authors: Berven.

\section{Correspondence}

Sigurd H. Berven, Department of Orthopaedic Surgery, University of California, San Francisco, 400 Parnassus Ave., 3rd Fl., San Francisco, CA 94143-0332. email: sigurd.berven@ucsf.edu. 